\title{
HIV in the United States and Dependent Areas
}

Of the 36,801 NEW HIV DIAGNOSES in the US and dependent areas* in 2019:

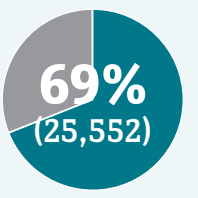

were among gay and bisexual men ${ }^{\dagger \ddagger}$

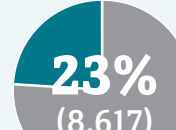

$(8,617)$ were among

heterosexuals ${ }^{* *}$
76

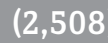

were among people who inject drugs ${ }^{\text {t+ }}$
Gay and bisexual men are the population most affected by HIV.

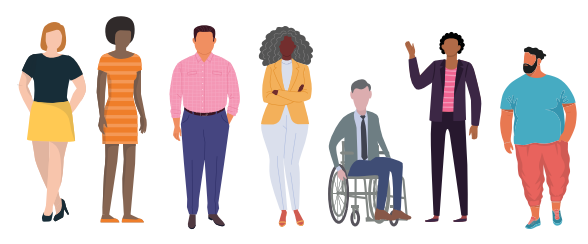

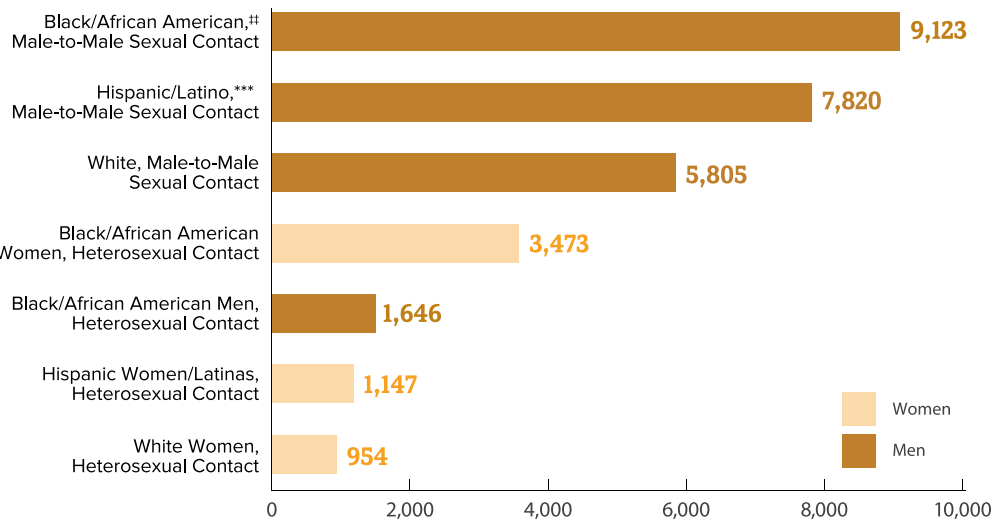

Subpopulations representing $2 \%$ or less of all people who received an HIV diagnosis in 2019 are not represented in this chart.

There are also differences in HIV diagnoses by age. People aged 13 to 34 made up more than half of all new HIV diagnoses in 2019.

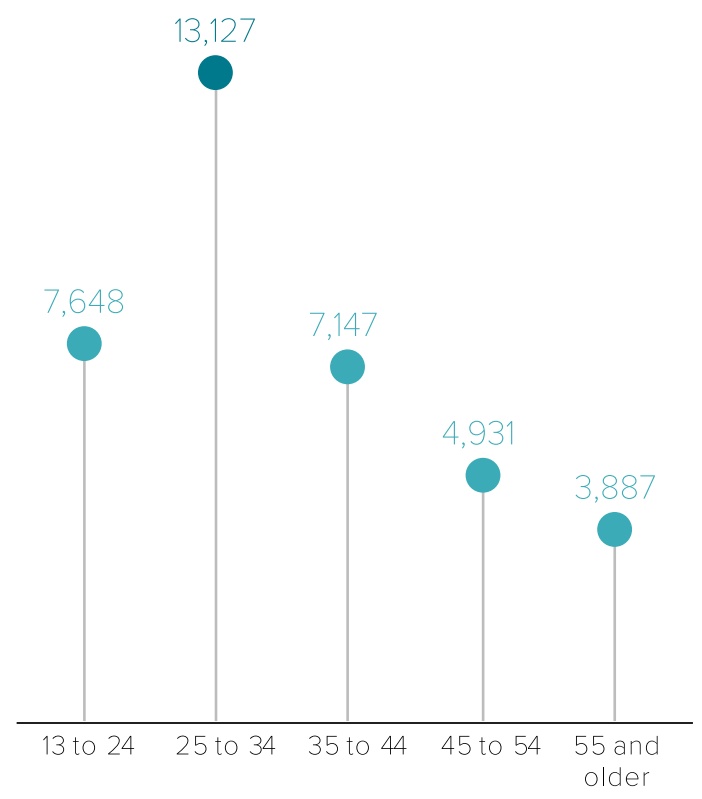

The number of new HIV diagnoses was highest among people aged 25 to 34 .

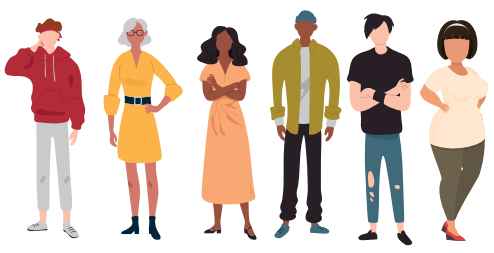




\section{From 2015 to 2019, HIV diagnoses decreased 9\% overall in the US and dependent areas.}

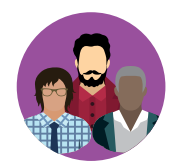

Gay and

bisexual men

$2015 \triangle 2019$

28,000

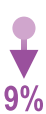

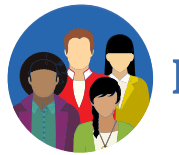

Heterosexuals

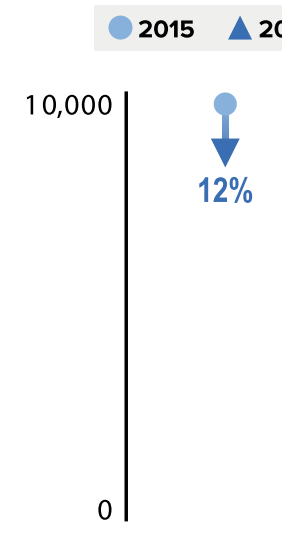

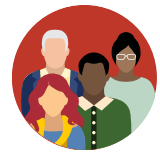

People who inject drugs

2015

$\triangle 2019$

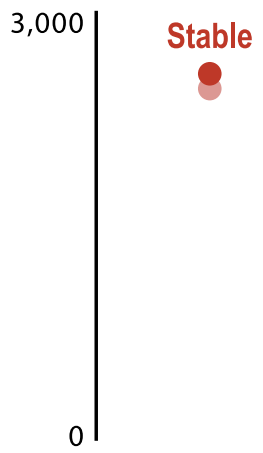

\section{Not all people with HIV are getting the care and treatment they need.}

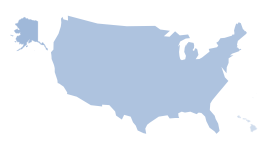

In 2019, an estimated $\mathbf{1 , 1 8 9 , 7 0 0 ~ P E O P L E ~ h a d ~ H I V . ~}{ }^{\dagger+}$

For every 100 people with HIV

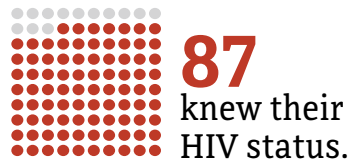

It is important for people to know their HIV status so they can take medicine to treat HIV if they have the virus. Taking HIV medicine every day can make the viral load undetectable. People who get and keep an undetectable viral load (or remain virally suppressed) can stay healthy for many years and will not transmit HIV to their sex partners.

Although more than half of adults and adolescents with diagnosed HIV are virally suppressed, more work is needed to increase these rates. For every 100 adults and adolescents with diagnosed HIV in 2019: ¥¥¥

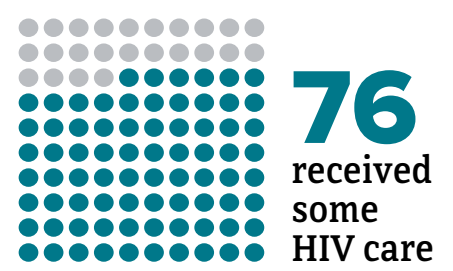

9000000000

000000000

000000000

0000909090

000000000

000000009

0000000000

0090909090

000090000

000000000

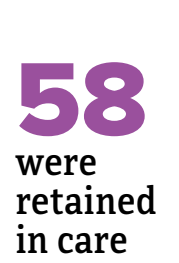

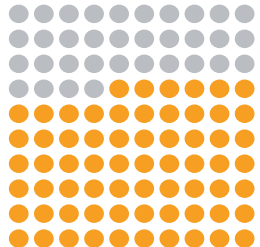

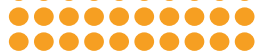

were virally suppressed

* American Samoa, Guam, the Northern Mariana Islands, Puerto Rico, the Republic of Palau, and the US Virgin Islands.

Includes infections attributed to male-to-male sexual contact and injection drug use (men who reported both risk factors).

₹ This fact sheet uses the term gay and bisexual men to represent gay, bisexual, and other men who reported male-to-male sexual contact.

** Does not include heterosexuals who reported injection drug use.

+t Does not include infections attributed to male-to-male sexual contact and injection drug use (men who reported both risk factors).

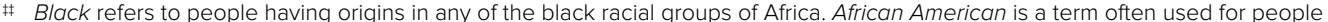

of African descent with ancestry in North America.

*** Hispanic/Latino people can be of any race.

t+t In 50 states and the District of Columbia.

\#¥ In 44 states and the District of Columbia.

For more information about HIV surveillance data, read the "Technical Notes" in the HIV surveillance reports at www.cdc.gov/hiv/library/reports/hiv-surveillance.html. 